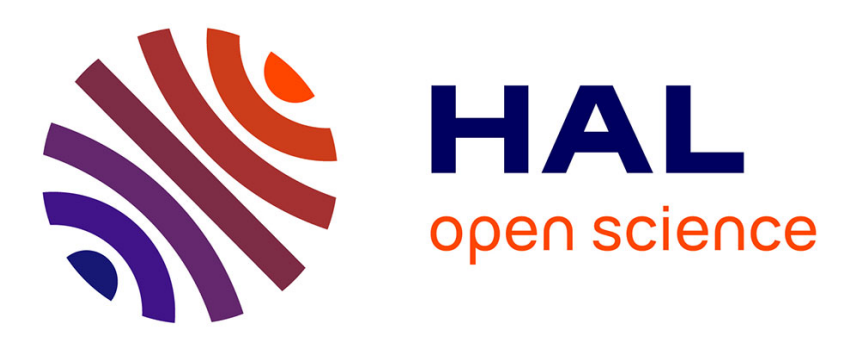

\title{
Détermination ultrasonore des propriétés élastiques et anélastiques en fonction de la fraction volumique des amas de Guinier-Preston dans des alliages aluminium-magnésium \\ C. Gault, A. Dauger, J. Wehr
}

\section{To cite this version:}

C. Gault, A. Dauger, J. Wehr. Détermination ultrasonore des propriétés élastiques et anélastiques en fonction de la fraction volumique des amas de Guinier-Preston dans des alliages aluminium-magnésium. Revue de Physique Appliquée, 1978, 13 (12), pp.853-858. 10.1051/rphysap:019780013012085300 . jpa-00244560

\author{
HAL Id: jpa-00244560 \\ https://hal.science/jpa-00244560
}

Submitted on 1 Jan 1978

HAL is a multi-disciplinary open access archive for the deposit and dissemination of scientific research documents, whether they are published or not. The documents may come from teaching and research institutions in France or abroad, or from public or private research centers.
L'archive ouverte pluridisciplinaire HAL, est destinée au dépôt et à la diffusion de documents scientifiques de niveau recherche, publiés ou non, émanant des établissements d'enseignement et de recherche français ou étrangers, des laboratoires publics ou privés. 


\title{
DÉTERMINATION ULTRASONORE DES PROPRIÉTÉS ÉLASTIQUES ET ANÉLASTIQUES EN FONCTION DE LA FRACTION VOLUMIQUE DES AMAS DE GUINIER-PRESTON DANS DES ALLIAGES ALUMINIUM-MAGNÉSIUM
}

\author{
C. GAULT \\ Laboratoire de Physique des Vibrations U.E.R. des Sciences Limoges, France \\ A. DAUGER \\ Laboratoire de Métallurgie Physique U.E.R. des Sciences Poitiers, France \\ J. WEHR $\dagger$ \\ Institut de Recherche Fondamentale Technologique Varsovie, Pologne
}

(Reçu le 30 juin 1978, accepté le 15 septembre 1978)

\begin{abstract}
Résumé. - Nous avons montré précédemment que la formation des amas de Guinier-Preston dans les alliages aluminium-magnésium s'accompagne d'une augmentation des vitesses de propagation des ultrasons et d'une diminution d'atténuation. Ce travail est consacré à l'explication quantitative de ces variations. On montre que la déformation du réseau cristallin de la matrice appauvrie provoque une diminution de la vitesse dans la matrice, et que l'augmentation mesurée doit s'expliquer par l'attribution aux amas de propriétés élastiques élevées. Dans le modèle utilisé l'augmentation relative de vitesse mesurée est proportionnelle à la fraction volumique occupée par les amas. Les variations d'atténuation sont interprétées en séparant l'atténuation due à la diffusion aux joints de grains et aux réflexions aux interfaces amas/matrice de celle due au frottement intérieur proprement dit. On montre qu'en fin d'évolution et aux fréquences considérées (6-15 MHz), seule cette dernière source est responsable de la diminution mesurée.
\end{abstract}

\begin{abstract}
It has been previously shown that the formation of Guinier-Preston zones in aluminium-magnesium alloys is accompanied by an increase of the velocities of ultrasonic waves, and a decrease of attenuation. This paper is an attempt to explain quantitatively these variations. It is shown that the coherence strain of the crystalline lattice of the depleted matrix induces a decrease of velocity in the matrix, and to explain the measured increase high elastic properties must be attributed to zones. The model used leads to an increase of velocity proportionnal to the fractional volume of the zones. To interprete the variations of attenuation the part due to grain scattering and reflection at the zone/matrix interfaces has been separated from that due to internal friction. It is shown that at the end of the evolution, and for the frequencies here considered $(6-15 \mathrm{MHz})$, the measured decrease of attenuation is only due to a decrease of internal friction.
\end{abstract}

1. Introduction. - $\mathrm{La}$ formation des amas de Guinier-Preston est le premier stade de précipitation d'une solution solide sursaturée aluminium-magnésium de teneur supérieure à $10 \%$ at. Les études cristallographiques par diffusion des rayons $\mathrm{X}$, microscopie électronique et diffraction des neutrons ont permis à Dauger et coll. $[1,2]$ de proposer le modèle suivant pour des amas formés à $20^{\circ} \mathrm{C}$ : sensiblement sphériques, de teneur $25 \%$ at. de magnésium, ordonnés de type $\mathrm{L}_{12}\left(\mathrm{Al}_{3} \mathrm{Mg}\right)$ et cohérents avec la matrice. La concentration de la matrice diminue de la concentration nominale de l'alliage jusqu'à $7,5 \%$ at. à l'équilibre. Les paramètres géométriques des amas ont été évalués, dans $\mathrm{Al}-\mathrm{Mg} 13 \%$ at. vieilli deux mois à $20^{\circ} \overline{\mathrm{C}}$ (le volume maximal des amas étant pratiquement atteint), en étudiant la diffusion des rayons $\mathrm{X}$ au voisinage des taches de Bragg [3] : diamètre $2 R=90 \AA$, écart à la cohérence $\eta=7 \times 10^{-3}$.

Nous avons montré récemment [4] que cette formation s'accompagne d'une augmentation des vitesses de propagation et d'une diminution de l'atténuation des ultrasons. Nous nous proposons ici d'analyser ces variations et de les relier quantitativement à la fraction volumique et aux paramètres géométriques des amas. 
2. Etude expérimentale. - Les mesures ont été effectuées par une méthode d'ondes ultrasonores pulsées de fréquence 6 et $15 \mathrm{MHz}$ [5]. Les vitesses de propagation ont été mesurées avec une précision d'environ $10^{-3}$, la sensibilité relative étant supérieure à $10^{-4}$. L'atténuation entre deux échos a été évaluée avec une précision de $\pm 0,1 \mathrm{~dB}$.

Les échantillons polycristallins de teneurs 13 et $16 \%$ (en atomes) de forme parallélépipédique (10 à $20 \mathrm{~mm}$ d'arête) ont subi un traitement de mise en solution solide à $450{ }^{\circ} \mathrm{C}$ suivi d'une trempe à l'air à $20{ }^{\circ} \mathrm{C}$. La variation de la vitesse de propagation des ultrasons en fonction du temps de vieillissement est portée par rapport à la valeur trouvée après réversion (Fig. 1). Ces variations sont indépendantes de la fréquence [6]. Sur la même figure ont été portées les variations d'atténuation au cours du même vieillissement (fréquences 6 et $15 \mathrm{MHz}$ ). L'atténuation diminue d'autant plus que la fréquence est plus élevée [4].

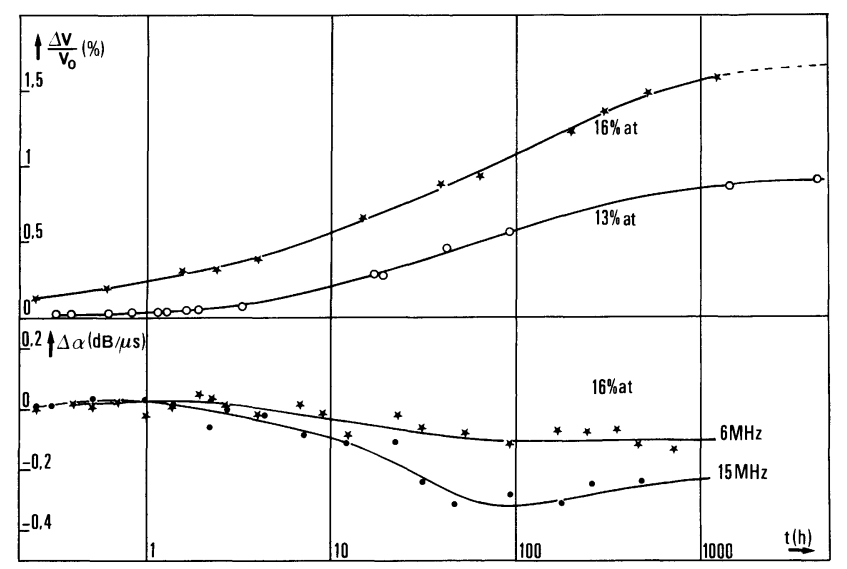

Fig. 1. - Vitesse de propagation et atténuation des ondes ultrasonores longitudinales au cours de vieillissements isothermes à $20{ }^{\circ} \mathrm{C}$ dans $\mathrm{Al}-\mathrm{Mg} 13 \%$ et $16 \%$ at.

[Velocity and attenuation of ultrasonic longitudinal waves during isothermal ageings at $20^{\circ} \mathrm{C}$ for $\mathrm{Al}-\mathrm{Mg} 13$ and 16 at. \%.]

3. Interprétation des résultats. - L'interprétation de ces mesures nécessite l'utilisation d'un modèle de propagation : on considère un milieu à deux phases (les amas G. P. et la matrice appauvrie) dont chacune a des propriétés homogènes vis-à-vis de la propagation des ultrasons. La très faible dimension des amas par rapport à la longueur d'onde ultrasonore $(R \sim 50 \AA, \lambda \sim 0,4 \mathrm{~mm}$ à $15 \mathrm{MHz})$ et la cohérence entre amas et matrice autorise à ne pas prendre en compte les déphasages dus aux réflexions multiples aux interfaces amas-matrice. Cette hypothèse est renforcée par l'indépendance des variations de vitesse vis-à-vis de la fréquence : la variation de module de Young mesurée à $70 \mathrm{kHz}$ en barreaux résonnants est la même que celle trouvée aux fréquences ultra- sonores [6]. Dans ce cas, la vitesse mesurée dans le matériau contenant les amas peut s'écrire [7] :

$$
V=\left[\frac{1-K}{V_{\mathrm{m}}}+\frac{K}{V_{\mathrm{z}}}\right]^{-1}
$$

où $V_{\mathrm{m}}$ est la vitesse de propagation dans la matrice appauvrie, et $V_{\mathrm{Z}}$ est un paramètre homogène à une vitesse qui traduit la propagation dans les amas G. P. $K$ est la fraction volumique occupée par les amas liée à la concentration atomique dans la matrice $k$, à la concentration atomique dans les amas $k_{\mathrm{z}}$ et à la concentration atomique initiale $k_{0}$ de l'alliage par :

$$
K \simeq \frac{k_{0}-k}{k_{\mathrm{z}}-k} .
$$

Par ailleurs, à cause de l'effet de taille important existant entre $\mathrm{Al}$ et $\mathrm{Mg}$, le réseau de la matrice subit des déformations locales dues à la cohérence et le paramètre cristallin de la matrice appauvrie varie en tout point. Le modèle de propagation proposé nécessite d'attribuer à la matrice des propriétés élastiques homogènes, ce qui conduit à attribuer un paramètre cristallin moyen $a_{\mathrm{m}}$ à la matrice (qui devra être évalué pour déterminer $V_{\mathrm{m}}$ ).

4. Détermination de la vitesse ultrasonore dans la matrice appauvrie. - Deux mécanismes interviennent lors de l'appauvrissement de la matrice :

- la diminution de la teneur en magnésium,

- la déformation du réseau cristallin autour des amas G. P. que l'on traduit par une modification de la maille moyenne de la matrice.

L'évaluation de ces deux effets nécessite la connaissance de la variation de la vitesse ultrasonore $V_{S}$ dans la solution solide $\mathrm{Al}-\mathrm{Mg}$ en fonction de la teneur $k$ et de la pression $P$. Ces grandeurs ont été obtenues à partir des mesures des constantes élastiques dans des monocristaux $[8,9]$ de teneurs comprises entre 4,5 et $12 \%$ at. de $\mathrm{Mg}$.

La variation de $V_{\mathrm{S}}$ avec $k$ est sensiblement linéaire autour de la teneur $7,5 \%$ et peut s'écrire [10] :

$$
V_{\mathbf{S}}=V_{\mathrm{s} 0}+\frac{\partial V_{\mathbf{s}}}{\partial k} k
$$

avec $V_{\mathrm{S} 0}=6390 \mathrm{~m} / \mathrm{s}$ et $\partial V_{\mathrm{S}} / \partial k=-380 \mathrm{~m} / \mathrm{s}$.

$\mathrm{La}$ variation de $V_{\mathrm{S}}$ due à une variation de paramètre cristallin a été évaluée à partir des mesures de constantes élastiques en fonction de la pression entre 1 et 2400 bars [10]. $\partial V_{\mathrm{S}} / \partial a$ dépend peu de la concentration en soluté :

$$
\begin{aligned}
& \frac{\partial V_{\mathrm{S}}}{\partial a}=-9,4 \times 10^{13} \mathrm{~s}^{-1} \text { pour l'aluminium pur } \\
& \frac{\partial V_{\mathrm{S}}}{\partial a}=-9,7 \times 10^{13} \mathrm{~s}^{-1} \text { pour } \mathrm{Al}-\mathrm{Mg} 12 \% \text { at }
\end{aligned}
$$


A partir d'un modèle d'amas sphériques assimilés à des inclusions élastiques dans un milieu isotrope fini [11, 12], on obtient [10] les relations suivantes, entre les paramètres cristallins moyens $a_{\mathrm{m}}$ dans la matrice appauvrie et $a_{\mathbf{z}}$ dans les amas, et celui $a_{\mathrm{S}}$ d'une solution solide de même teneur que la matrice appauvrie :

$a_{\mathrm{m}}=a_{\mathrm{S}}\left[1+\eta(1+\delta) \frac{K}{1-K}\right]\left[1-\eta \frac{K}{1-K}\right]$

$a_{\mathrm{Z}}=a_{\mathrm{S}}\left[1+\eta(1+\delta) \frac{K}{1-K}\right][1+\eta]$

avec $\delta=\frac{4}{3} \frac{G}{B}, G$ et $B$ étant les modules de cisaillement et de rigidité moyens du milieu.

L'écart à la cohérence $\eta$ s'exprime par la relation approchée :

$$
\eta \# \frac{4}{3}(1-K) \frac{a_{\mathrm{z}}^{\prime}-a_{\mathrm{s}}}{a_{\mathrm{z}}^{\prime}+a_{\mathrm{s}}}
$$

où $a_{\mathrm{Z}}^{\prime}$ est le paramètre cristallin d'un amas sans contrainte. A partir des résultats obtenus pour $\eta$ en diffusion des rayons $\mathrm{X}$ [3], $a_{\mathrm{Z}}^{\prime}$ a pu être évalué $\left(a_{\mathrm{Z}}^{\prime}=4,14 \AA\right) ; a_{\mathrm{S}}$ est connu expérimentalement

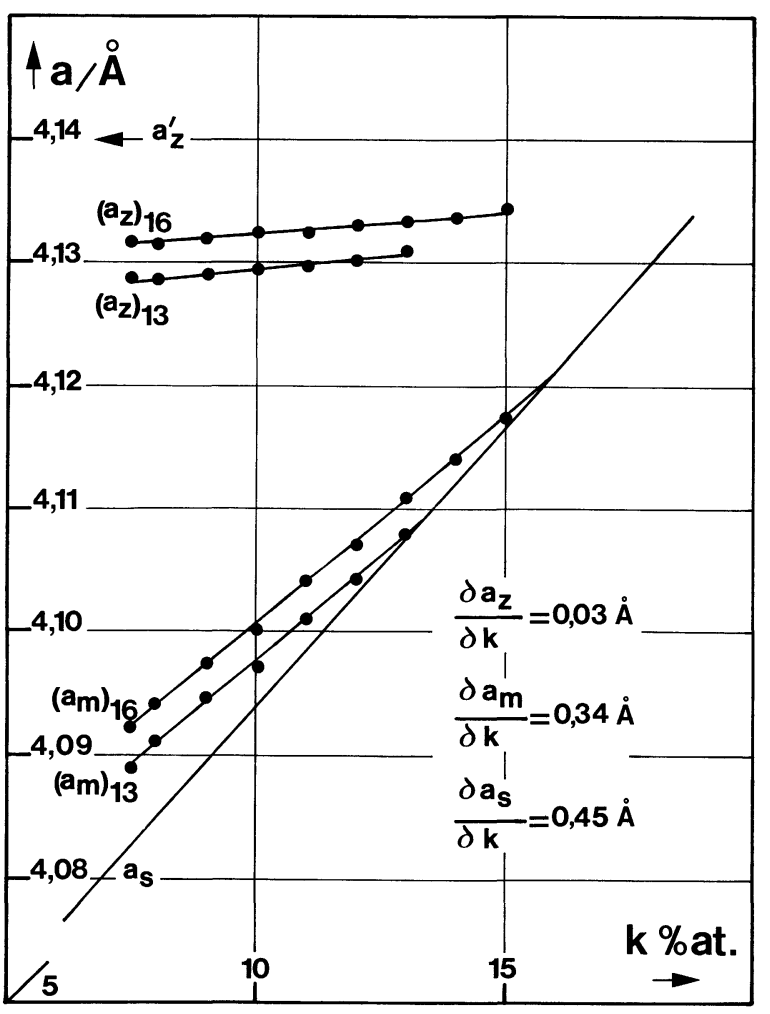

FIG. 2. - Variation des paramètres cristallins $a_{\mathrm{Z}}$ et $a_{\mathrm{m}}$ dans les amas et dans la matrice avec la teneur en magnésium $k$ dans la matrice appauvrie. $a_{\mathrm{s}}$ est le paramètre cristallin d'une solution solide de même teneur que la matrice appauvrie.

[Variation of the lattice parameters $a_{\mathrm{z}}$ and $a_{\mathrm{m}}$ of the zones and of the matrix with the magnesium concentration $k$ in the depleted matrix, $a_{\mathrm{S}}$ is the lattice parameter of a solid solution of the same concentration that the depleted matrix.] pour la solution solide sursaturée Al-Mg [13]; $\eta$, donc $a_{\mathrm{m}}$ et $a_{\mathrm{z}}$ peuvent alors être déterminés en fonction de $K$ ou de $k$ (cf. relation (2)). La figure 2 donne les résultats de ce calcul pour $\mathrm{Al}-\mathrm{Mg} 13$ et $16 \%$.

Il convient de noter que le modèle utilisé suppose implicitement que $a_{\mathbf{z}}^{\prime}$ est constant vis-à-vis de $k$, c'est-à-dire qu'au cours de leur développement, les amas restent ordonnés et gardent la même composition, dans une matrice qui s'appauvrit progressivement. Cette hypothèse est conforme à l'évolution dissymétrique des maximums d'intensité diffusée en rayons $\mathrm{X}$ qui s'explique par un grossissement des particules ordonnées dont le nombre et la composition ne varient pas [2].

Compte tenu de ces résultats et sachant que la vitesse de propagation dans la matrice appauvrie peut s'écrire $V_{\mathrm{m}}=V_{\mathrm{S}}+(\Delta V)_{\text {maille }}$ il vient :

$$
V_{\mathrm{m}}=V_{0}+\left[\frac{\partial V_{\mathrm{S}}}{\partial a}\left(\frac{\partial a_{\mathrm{s}}}{\partial k}-\frac{\partial a_{\mathrm{m}}}{\partial k}\right)-\frac{\partial V_{\mathrm{s}}}{\partial k}\right]\left[k_{0}-k\right]
$$

où $V_{0}$ est la vitesse dans la solution solide sursaturée instable initiale.

A partir des résultats numériques de (3), (4) et figure 2, la relation (8) permet de calculer les variations de $V_{\mathrm{m}}$, portées sur la figure 3. L'appauvrissement de la matrice entraîne une diminution de $V_{\mathrm{m}}$, cet effet provenant essentiellement du champ de déformation dû à la présence des amas, car la diminution de concentration en magnésium seule tendrait à faire augmenter la vitesse.

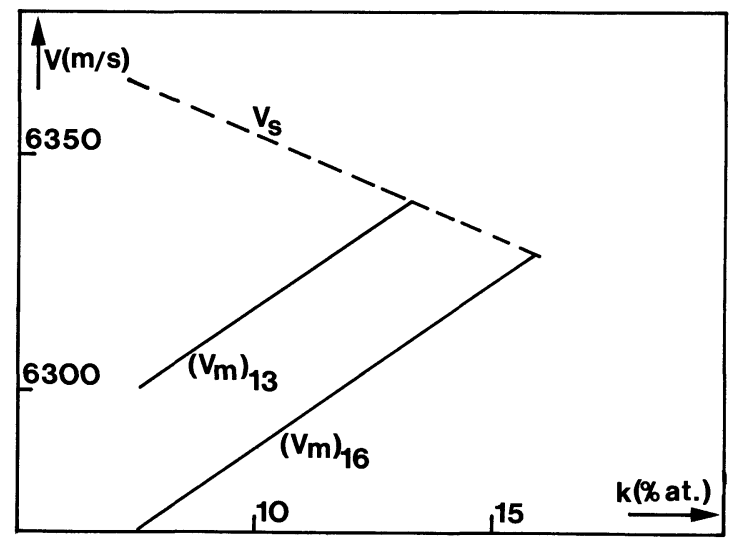

FIG. 3. - Vitesse de propagation dans la matrice appauvrie pour $\mathrm{Al}-\mathrm{Mg} 13$ et $16 \%$.

[Ultrasonic velocity in the depleted matrix for $\mathrm{Al}-\mathrm{Mg} 13$ and $16^{\circ}{ }_{0}$.]

L'augmentation de vitesse mesurée au cours du développement des amas ne peut donc provenir que d'un effet propre aux amas G. P., et ne peut s'expliquer qu'en leur attribuant des vitesses de propagation (donc des constantes élastiques) élevées.

5. Relation entre $\Delta V / V_{0}$ et $K$. $-V_{\mathrm{m}}$ étant connue on peut, à partir de la relation (1) et des valeurs 
asymptotiques des courbes expérimentales de la figure 1 pour lesquelles les fractions volumiques maximales sont atteintes, calculer la valeur de $V_{\mathrm{Z}}$ qui doit être attribuée aux amas G. P. Les résultats sont donnés dans le tableau I.

\section{TABLEAU I}

$\begin{array}{ccccc}k_{0} & K & V_{0} & \frac{\Delta V}{V_{0}} & V_{\mathrm{Z}} \\ - & - & - & - & - \\ 13,3 \% & 0,331 & 6340 \mathrm{~m} / \mathrm{s} & 1 \% & 6620 \mathrm{~m} / \mathrm{s} \\ 16 \% & 0,490 & 6329 \mathrm{~m} / \mathrm{s} & 1,65 \% & 6610 \mathrm{~m} / \mathrm{s}\end{array}$

Les valeurs trouvées pour $V_{\mathrm{Z}}$ paraissent élevées par rapport à celles usuelles dans les alliages $\mathrm{Al}-\mathrm{Mg}$. Mais on peut noter que $a_{\mathrm{Z}}^{\prime}$ dans les amas sans contrainte est inférieur au paramètre d'une solution solide désordonnée contenant $25 \%$ at. de magnésium [13], cet effet pouvant être une conséquence de l'ordre interne. Cette diminution de maille pourrait à elle seule expliquer les fortes valeurs trouvées pour $V_{\mathbf{z}}$.

L'ordre de grandeur de $V_{\mathrm{Z}}$ étant connu, la relation (1) rend compte de la relation entre $\Delta V / V_{0}$ et $K$ qui peut s'écrire, en utilisant les relations (2) et $(8)$ :

$$
\frac{\Delta V}{V_{0}}=\left(\frac{V_{\mathrm{z}}-V_{0}}{V_{\mathrm{z}}}+\frac{k_{\mathrm{z}}-k_{0}}{V_{0}} b\right) K
$$

avec

$$
b=\frac{\partial V_{\mathbf{s}}}{\partial a}\left(\frac{\partial a_{\mathrm{s}}}{\partial k}-\frac{\partial a_{\mathrm{m}}}{\partial k}\right)-\frac{\partial V_{\mathrm{s}}}{\partial k} .
$$

Cette expression permet, connaissant $V_{\mathrm{Z}}$, de calculer le rapport entre $\Delta V / V_{0}$ et $K$ pour un alliage donné. Ce rapport n'est pas exactement constant car $V_{\mathrm{Z}}$ dépend de $K$ puisque la maille diminue légèrement dans les amas au fur et à mesure de leur développement. Cependant la figure 2 montre que cette diminution est faible (de l'ordre de $0,002 \AA$ ) et $V_{\mathrm{Z}}$ peut être considérée comme sensiblement constante vis-à-vis de $K$. Compte tenù de cette approximation et du modèle utilisé, $\Delta V / V_{0}$ est donc sensiblement proportionnel à $K$, tout au moins pour les stades ultimes de développement des amas. La relation (9) montre qu'à $20^{\circ} \mathrm{C}$ :

$$
\frac{\Delta V}{V_{0}} \sim\left[3 \times 10^{-2}\right] \mathrm{K} .
$$

6. Analyse des variations d'atténuation. - Les mécanismes susceptibles de provoquer ces variations d'atténuation sont : la diffusion aux joints de grains des polycristaux, l'atténuation due aux changements d'impédance acoustique entre les amas et la matrice appauvrie, l'atténuation intrinsèque dans les amas et dans la matrice (frottement intérieur).
6.1 Diffusion AUX JoINTS DE GRAINS. - Le diamètre moyen des grains $\bar{D}$ étant de l'ordre de $0,25 \mathrm{~mm}$, la longueur d'onde est inférieure à $2 \Pi \bar{D}$ ce qui correspond au domaine de diffusion intermédiaire où l'atténuation est proportionnelle au carré de la fréquence [14] :

$$
\alpha_{\mathrm{g}}=A f^{2}
$$

$A$ est un coefficient qui dépend des paramètres géométriques des grains et de leurs propriétés élastiques. La formation des amas G. P. a donc pour conséquence de faire varier $A$. A partir des expressions données par [14] pour $A$, et de nos résultats expérimentaux, nous avons établi [10] :

$$
\frac{\Delta A}{A} \# 6 \frac{\Delta V}{V_{0}} \text { pour Al-Mg } 16 \%
$$

$\alpha_{\mathrm{g}}$ augmente donc avec la formation des amas puisque $V$ augmente. Cependant l'évaluation quantitative de $A$ est délicate théoriquement, et on ne peut espérer calculer $\Delta \alpha_{\mathrm{g}}$ à partir de $\Delta \alpha_{\mathrm{g}} / \alpha_{\mathrm{g}}$.

6.2 RÉFlEXION AUX INTERFACES AMAS/MATRICE. $\mathrm{Si}$ on néglige les phénomènes de diffusion multiple aux interfaces, on peut considérer les amas comme des inclusions assimilables à des couches minces pour lesquelles le coefficient de transmission de l'onde ultrasonore vaut [7] :

$$
q=\frac{\left(1-\sigma^{2}\right) \exp \left(-i \frac{2 \Pi}{i} l\right)}{1-x^{2} \sigma^{2} \exp \left(-i \frac{4 \Pi}{i} l\right)}
$$

$x$ est un coefficient de forme qui tient compte de la géométrie des interfaces $(x \leqslant 1, x=1$ pour des interfaces planes).

$\sigma$ est le coefficient de réflexion entre amas et matrice :

$$
\sigma=\frac{\rho_{\mathrm{m}} V_{\mathrm{m}}-\rho_{\mathrm{Z}} V_{\mathrm{Z}}}{\rho_{\mathrm{m}} V_{\mathrm{m}}+\rho_{\mathrm{Z}} V_{\mathrm{Z}}}
$$

$\rho_{\mathrm{Z}}$ et $\rho_{\mathrm{m}}$ étant les masses volumiques des amas et de la matrice.

Le coefficient de réflexion énergétique s'écrit alors pour $2 \Pi l / \lambda \ll 1$ et $\sigma \ll 1$ :

$1-|q|^{2} \# 2\left[\sigma^{2}\left(1-x^{2}\right)+2 \sigma^{2} x^{2}\left(\frac{2 \Pi l}{\lambda}\right)^{2}\right]$

On montre que si le nombre d'amas est suffisamment élevé, le coefficient d'atténuation $\alpha_{R}$ dû aux réflexions aux interfaces est tel que

$$
1-|q|^{2} \# 2 \alpha_{\mathrm{R}} \Delta l,
$$

où $\Delta l$ est le libre parcours moyen de l'onde dans la matrice [7]. D'où l'expression pour $\alpha_{R}$ :

$$
\alpha_{\mathbf{R}} \# \frac{\sigma^{2}}{\Delta l}\left[\left(1-x^{2}\right)+2 x^{2}\left(\frac{2 \Pi l}{i}\right)^{2}\right] \text {. }
$$

Si $R$ est le rayon d'un amas, $l=2 R$ et $\Delta l=2 R / K$. 
Par ailleurs, $R_{0}$, le rayon de la sphère d'influence d'un amas est défini par : $K=\left(R / R_{0}\right)^{3}$. En substituant dans (15) et en écrivant que $\lambda=V_{\mathrm{Z}} \mid f$, on obtient :

$$
\alpha_{\mathrm{R}}=\frac{1-x^{2}}{2 R_{0}} \sigma^{2} K^{2 / 3}+\frac{8 x^{2} \Pi^{2} \sigma^{2} R_{0} K^{4 / 3}}{V_{\mathrm{Z}}^{2}} f^{2}
$$

En prenant des valeurs maximales $(x=1$; $\sigma=1,2 \times 10^{-4} ; R_{0}=100 \AA ; K=0,5 ; V_{\mathrm{Z}}=6600 \mathrm{~m} / \mathrm{s}$; $f=15 \mathrm{MHz}$ ) on constate que le deuxième terme de la relation (16) est négligeable (de l'ordre de $\left.10^{-5} \mathrm{~dB} / \mu \mathrm{s}\right)$ ce qui permet d'écrire l'expression simplifiée :

$$
\alpha_{\mathrm{R}} \# \frac{1-x^{2}}{2 R_{0}} \sigma^{2} K^{2 / 3}
$$

$\sigma^{2} K^{2 / 3}$ est calculé numériquement dans le cas d'Al-Mg $16 \%$ sur la figure 4 , et représente $\alpha_{R}$ au facteur $\frac{1-x^{2}}{2 R_{0}}$ près (qui n'est pas obligatoirement constant avec $K$ et dépend vraisemblablement de la fréquence). Cependant cette courbe montre que $\alpha_{\mathrm{R}}$ contribue à augmenter l'atténuation pendant la formation des amas G. P., mais que sa contribution devient négligeable en fin d'évolution.

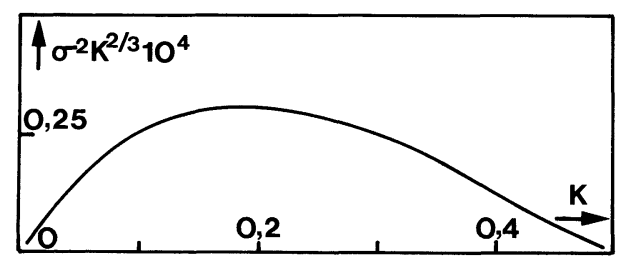

FIG. 4. - Aspect de la variation d'atténuation due aux réflexions aux interfaces amas/matrice pour $\mathrm{Al}-\mathrm{Mg} 16 \%$ en fonction de la fraction volumique occupée pour les amas.

[Aspect of the variation of attenuation due to reflections at the zone/matrix interfaces for $\mathrm{Al}-\mathrm{Mg} 16 \%$ versus the fractional volume of zones.]

6.3 VARIATION DE FROTTEMENT INTÉRIEUR. L'atténuation due au frottement intérieur est la différence entre l'atténuation mesurée et les termes dus aux réflexions (aux joints de grains et au niveau des amas) :

$$
\Delta \alpha_{Q-1}=\Delta \alpha-\Delta \alpha_{\mathrm{g}}-\alpha_{\mathrm{R}}
$$

$\Delta \alpha$ étant négatif et $\Delta \alpha_{g}$ et $\alpha_{R}$ positifs, la formation des amas G. P. entraîne donc une diminution de frottement intérieur. L'évaluation quantitative de cette diminution est impossible en général. Cepen- dant, en fin d'évolution $\alpha_{\mathrm{R}}$ est négligeable et on peut alors écrire $\Delta \alpha=\Delta \alpha_{Q^{-1}}+\Delta \alpha_{g}$. Sachant que

$$
\begin{gathered}
\alpha_{Q^{-1}}=27,3 Q^{-1} f \quad[10] \\
\Delta \alpha=27,3 \Delta Q^{-1} f+\Delta A f^{2} .
\end{gathered}
$$

Dans le cas d'Al-Mg et pour le domaine de fréquence (6-15 $\mathrm{MHz})$ considéré, on peut admettre que $Q^{-1}$, donc $\Delta Q^{-1}$, varie peu avec la fréquence [4]. L'écriture de la relation (18) pour deux fréquences $f_{1}$ et $f_{2}$ où les variations mesurées sont $\Delta \alpha_{1}$ et $\Delta \alpha_{2}$, donne :

$$
\begin{aligned}
\Delta Q^{-1} & =\frac{1}{27,3}\left[\frac{f_{2}}{f_{1}\left(f_{2}-f_{1}\right)} \Delta \alpha_{1}-\frac{f_{1}}{f_{2}\left(f_{2}-f_{1}\right)} \Delta \alpha_{2}\right] \\
\Delta A & =\frac{1}{f_{2}\left(f_{2}-f_{1}\right)} \Delta \alpha_{2}-\frac{1}{f_{1}\left(f_{2}-f_{1}\right)} \Delta \alpha_{1} .
\end{aligned}
$$

Pour Al-Mg $16 \%, f_{1}=6 \mathrm{MHz}, f_{2}=15 \mathrm{MHz}$, $\Delta \alpha_{1}=0,11 \mathrm{~dB} / \mu \mathrm{s}, \Delta \alpha_{2}=-0,26 \mathrm{~dB} / \mu \mathrm{s}$, on trouve :

$$
\Delta Q^{-1}=-7 \times 10^{-4}, \Delta A=10^{-4} \mathrm{~dB} / \mathrm{MHz},
$$
soit

$$
\Delta \alpha_{\mathrm{g}}=0,02 \mathrm{~dB} / \mu \mathrm{s} \text { à } 15 \mathrm{MHz} .
$$

Ces résultats montrent que la variation d'atténuation due à la diffusion aux joints de grains est très faible et, qu'en fin d'évolution, la variation mesurée est pratiquement celle due au frottement intérieur.

7. Conclusion. - L'analyse de l'effet d'appauvrissement de la matrice sur les vitesses de propagation ultrasonores a permis de montrer que le champ de déformation autour des amas a une influence prépondérante. Compte tenu du modèle utilisé, l'augmentation de vitesse de propagation mesurée peut être considérée comme proportionnelle à la fraction volumique occupée par les amas.

L'interprétation de la diminution d'atténuation a été faite en considérant les trois mécanismes, diffusion aux joints de grains, réflexion aux interfaces amasmatrice, frottement intérieur. Nous avons montré qu'en fin d'évolution la diminution mesurée est pratiquement due au seul frottement intérieur. La relation simple établie entre la vitesse de propagation des ultrasons et la fraction volumique occupée par les amas G. P. montre que ce type de mesure est bien adapté à l'étude des cinétiques de croissance des amas G. P. dans Al-Mg [10].

Remerciements. - Les auteurs français tiennent à saluer avec émotion la mémoire du Pr. J. Wehr tragiquement disparu en 1977 et à rendre hommage à ses éminentes qualités scientifiques et humaines. 


\section{Bibliographie}

[1] Dauger, A., Boudili, E. K., Roth, M., Scr. Metall. 10 (1976) 1119.

[2] Boudili, E. K., Denanot, M. F., Dauger, A., Scr. Metall. $11(1977) 543$.

[3] Dauger, A., Guillot, J. P., Caisso, J., Acta Metall. 22 (1974) 733.

[4] Gault, C., Dauger, A., Boch, P., Il Nuovo Cimento 33B (1976) 345.

[5] Gault, C., Vergnol, J., Dauger, A., Villain, J. P., Boch, P., Mém. Sci. Rev. Métall. 12 (1975) 921.

[6] Gault, C., Dauger, A., Boch, P., Phys. Status Solidi (a) 31 (1975) 179.

[7] WeHR, J., Mesure de la vitesse et de l'atténuation des ondes ultrasonores (en polonais), P.W.N. (Varsovie 1972).
[8] Gault, C., Dauger, A., Boch, P., Proceedings of the Spring Conference of the Institute of Acoustics of Bath (1977) 6-9-1.

[9] Gault, C., Dauger, A., Boch, P., Phys. Status Solidi (a) 43 (1977) 625.

[10] Gault, C., Thèse de Doctorat ès Sciences Limoges (1978).

[11] Ashby, Brown, Phil. Mag. 8 (1963) 1083.

[12] QuÉRÉ, Y., Défauts ponctuels dans les métaux (Masson, Paris) 1967.

[13] Luo, H. L., Chao, C. C., Duwez, P., Trans. Met. Soc. AIME $230(1964) 1488$.

[14] Papadakis, E. P., Physical Acoustics, Vol. 4, Part B, New York, N.Y. (1968) 269. 